\title{
Laser Cleaning Technique Using Laser-induced Acoustic Streaming for Silicon Wafers
}

\author{
Chwan-Huei Tsai and Wen-Shiang Peng \\ *Department of Mechatronic Engineering, Huafan University, No. 1, Huafan Rd., Shihding District, \\ New Taipei City 22301, Taiwan \\ E-mail: chtsai@cc.hfu.edu.tw
}

\begin{abstract}
This paper proposes an alternative laser cleaning technique for silicon wafers to overcome the difficulties in removing submicron particles by using the conventional ultrasonic cleaning method. An Nd:YAG laser was applied on the back side of a wafer submerged in water. The laser energy induced shock waves in the wafer, which were transmitted into the water and a streaming bubble flow was generated. The shock waves and bubble flow removed the alumina particles of $0.5 \mu \mathrm{m}$ in size from the wafer surface. Because the wafer was in contact with the water, the damage caused by the laser heat was significantly reduced. This study analyzed the laser stationary and laser scan cleaning techniques and established the laser power and scanning speed parameters. The cleaning efficiency of the proposed technique was found to be excellent, and the laser power required was less than 50 $\mathrm{W}$. The temperature increase in the wafer caused by the laser heating was less than $30^{\circ} \mathrm{C}$. Thus, the proposed acoustic streaming technique has great potential to provide an improved solution for silicon wafer cleaning.
\end{abstract}

DOI: 10.2961/jlmn.2017.01.0001

Keywords: laser cleaning, acoustic streaming, bubble flow, particle removal, silicon wafers

\section{Introduction}

The conventional ultrasonic silicon wafer cleaning method is based on the acoustic streaming theory. Steady vortices or circulations are generated because of the acoustic waves in fluids [1] and a turbulent jet is induced from the acoustic wave [2]. A high intensity sound wave can induce fluid motion. The acoustic streaming theory has been applied in the process of biofouling removal [3]. The conventional acoustic wave generation methods involve creating ultrasonic waves by using piezoelectric devices to induce surface acoustic waves. The Van der Waals force rapidly increases with the decrease in particle size. Conventional ultrasonic cleaning methods cause difficulties in removing submicron particles. The smaller the particles are, the higher the sonic frequency must be. Particle sizes of less than $100 \mathrm{~nm}$ have been successfully removed using megasonic cleaning with high frequency megahertz sonic energy [4].

Laser cleaning by using a pulsed light to induce the removal of contamination from a surface has great potential. Two types of laser cleaning techniques have been widely studied: dry laser cleaning [5-7] and steam laser cleaning [8-10]. In dry laser cleaning, the laser evaporates the particles to induce a large cleaning force greater than the adhesion force between the particles and substrate. The dry laser cleaning technique has been applied to the surface cleaning of silicon wafers; the force resulting from the surface acceleration, exceeding the van der Waals adhesion force, causes the particle to be removed from the surface [5].

In steam laser cleaning, a liquid film is placed on the substrate surface. The laser energy induces the explosive evaporation of the liquid, causing the removal of the contaminants. The resulting explosive vaporization of the ad- sorbed water molecules generates forces larger than the adhesion forces between the particle and the substrate, which propels the particles off the surface.

In dry laser cleaning, particular attention has been focused on damage-free particle removal. The laser beam directly irradiates onto the substrate to remove the particles and the particles absorb the laser energy and melt. The hot particles damage the substrate surface. To reduce the damage resulting from the thermal contact with hightemperature particles, steep-front short-duration pulses are necessary [11]. The dynamics of the particle ejection from the substrate was studied to elucidate the ablation mechanism involved in laser dry cleaning [12].

An alternative dry cleaning method by using laser shock waves has been demonstrated to remove the submicron particles from solid surfaces [13-16]. In this method, the laser beam direction is parallel to the substrate, and a gap exists between the laser beam and substrate. The cleaning efficiency depends on the gap between the initiation position of the plasma shock waves and silicon wafer surface [16]. For smaller gaps, the removal efficiency is high.

In dry laser silicon wafer cleaning, the laser energy may damage the surface by melting the particles or plasma shock waves. In general, steam cleaning reduces the thermal effect on the silicon wafer surface. Mosbacher et al. [17] observed that the threshold of steam cleaning for various particle sizes and materials is universal. The threshold coincides with the laser energy necessary for the onset of explosive vaporization [18]. However, the near-field focusing of the beam may cause defects on the silicon surface in laser steam cleaning $[18,19]$.

The abovementioned references are frontal cleaning methods in which the laser radiates on the desired cleaning 
surface. Another cleaning technique known as backside cleaning has also been studied [20-22]. In this technique, the particles were removed by laser irradiation on the rear side of the wafers. Leiderer et al. [20] proposed the backside cleaning of silicon wafers in their study and removed submicron particles of $170 \mathrm{~nm}$ by using an Nd:YAG laser applied to the backside of silicon wafers. The particles were detached by an acoustic shock wave traveling to the front side of the wafers [20].

An alternative laser cleaning technique, laser-induced acoustic streaming, is proposed in this study. In this technique, the laser is irradiated on the back side of the wafers submerged in water, which induces an acoustic stream generating a turbulent bubble flow that cleans the surface.

Unlike the previously studied [20-22] dry laser cleaning that uses the shock wave induced by the laser to remove the particles from the surface, the particle removal mechanism in this study involved the water streaming bubble flow generated by the shock wave in the wafer and water. This particle removal mechanism is completely distinct from the aforementioned conventional laser cleaning. This innovative approach offers great potential for efficient laser cleaning.

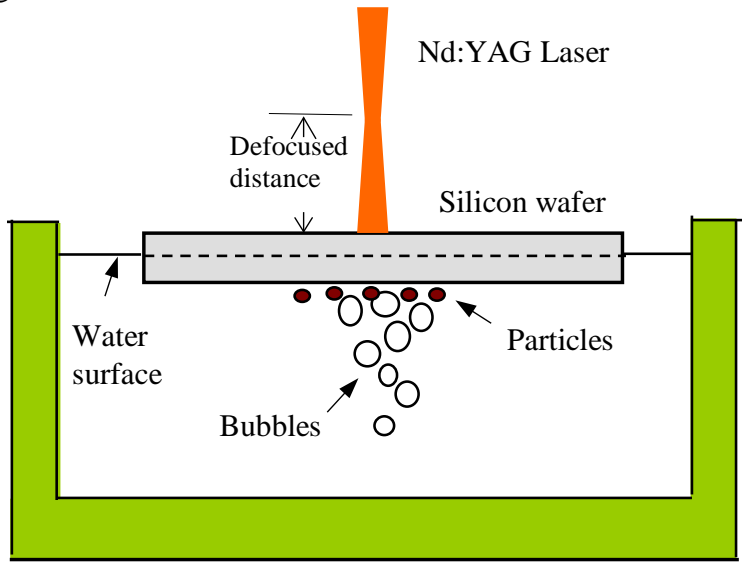

Fig. 1 Configuration of the laser cleaning technique using acoustic streaming

\section{Principle of laser-induced acoustic streaming}

\subsection{Laser cleaning system and substrates}

Figure 1 illustrates the configuration of the laser cleaning system. In this study, the substrate was placed in water. The desired cleaning surface was in contact with water, and the laser was applied from the back side of the silicon wafer. A pulsed Nd:YAG laser was used in this study. An unpolarized laser beam was used, and the beam profile was Gaussian mode TEM $_{00}$. The orientation of the silicon wafer used was $\{111\}$, and the thickness was $220 \mu \mathrm{m}$. The contaminating particles attached to the wafer surface were alumina particles of size $0.5 \mu \mathrm{m}$. Alumina particles and pure water were mixed in a weight ratio of $1: 10$. The mixture was then poured onto the wafer surface uniformly and heated at $60^{\circ} \mathrm{C}$ for $3 \mathrm{~h}$.

The wavelength of the Nd:YAG laser was $1064 \mathrm{~nm}$. The focal length of the objective lens was $80 \mathrm{~mm}$, and the minimum diameter of the focused spot was $32 \mu \mathrm{m}$. To prevent thermal damage, the laser focal plane was placed above the wafer surface at a defocused distance of $40 \mathrm{~mm}$ and a laser spot diameter of $1.69 \mathrm{~mm}$. Because the laser was applied to the back side of the substrate, the damage caused by the laser heat could be substantially reduced.

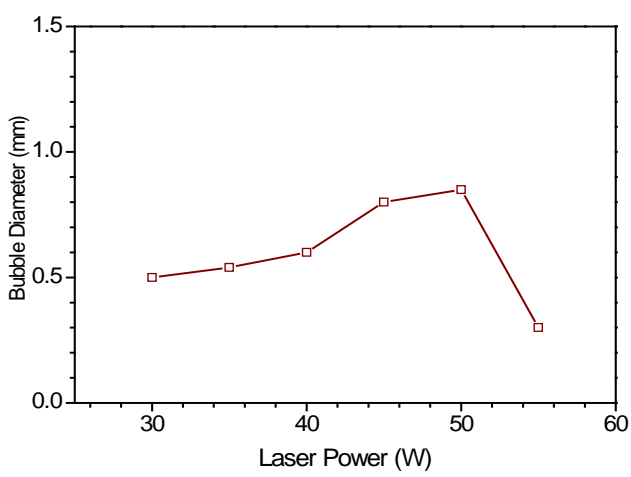

Fig. 2 Bubble diameter for different laser power at the laser repetition rate of $20 \mathrm{kHz}$.

\subsection{Acoustic streaming and bubble flow}

The laser applied on the silicon surface generates shock waves, which propagate across the water and generate an acoustic streaming bubble flow. The bubbles move rapidly with turbulence. The bubble size and the streaming type were affected by the laser power and repetition rate.

At a $40 \mathrm{~W}$ laser power, the threshold of the laser repetition rate of bubble initiation was approximately $5 \mathrm{kHz}$. When the laser repetition rate was increased beyond 20 $\mathrm{kHz}$, the bubble size grew to a stable size of approximately $0.6 \mathrm{~mm}$ diameter. The images of bubbles were captured using a high-speed camera. Figure 2 represents the relationship between the bubble size and laser power at a repetition rate of $20 \mathrm{kHz}$. At a $30 \mathrm{~W}$ laser power, the initiated bubble was small with a diameter of $0.3 \mathrm{~mm}$. When two small bubbles moved close together, the bubbles fused to form a larger bubble with a median diameter of $0.5 \mathrm{~mm}$. The higher the applied laser power was, the larger the bubble was. However, when the laser power was more than $50 \mathrm{~W}$, the bubbles generated and collapsed rapidly and had no time to combine together before collapsing. At a laser power of 55 $\mathrm{W}$, the bubble size retained the small size of $0.3 \mathrm{~mm}$ in diameter

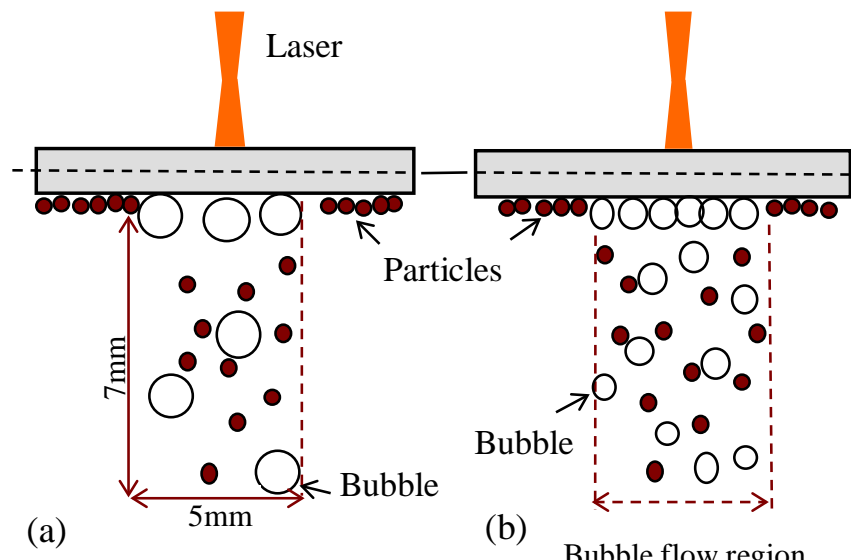

Bubble flow region

Fig. 3 Configuration of bubble flow and acoustic streaming for the laser power of (a) $30 \mathrm{~W}$ and (b) $55 \mathrm{~W}$. 


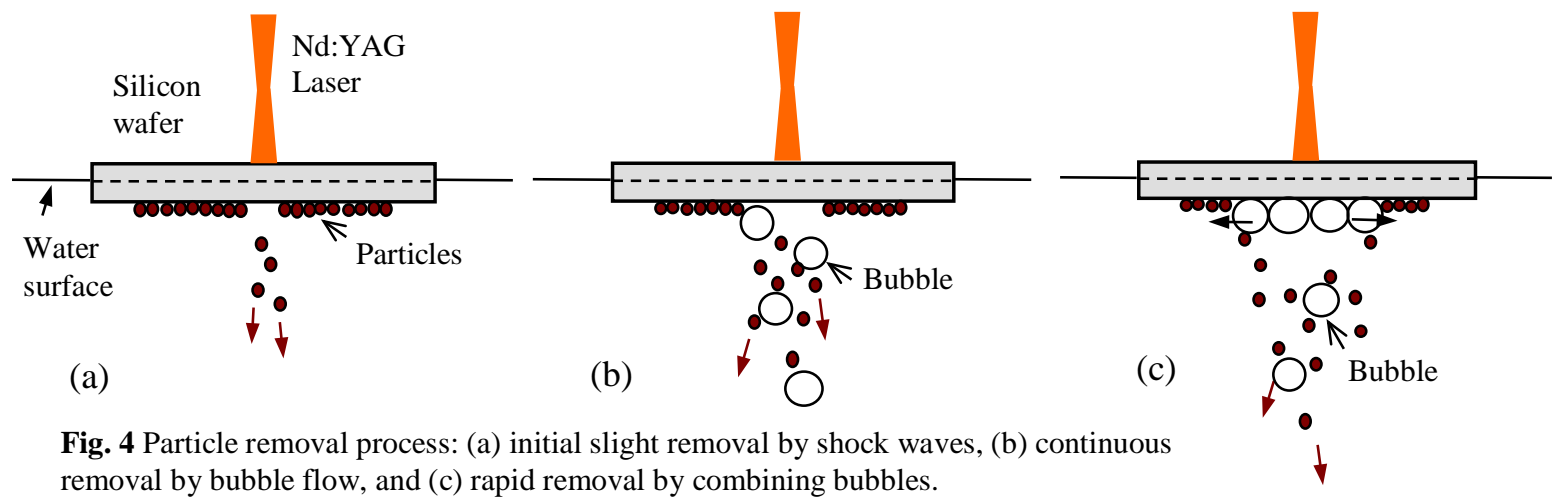

Figure 3(a) and 3(b) present the configurations of acoustic streaming under a confined bubble flow region for the laser powers of 30 and $55 \mathrm{~W}$ at a repetition rate of 20 $\mathrm{kHz}$, respectively. The dark red circles depict the particles and the white circles depict the bubbles. The bubble flow region is a cylindrical volume of depth and diameter of approximately 7 and $5 \mathrm{~mm}$, respectively. The range of the bubble flow region is independent of the laser repetition rate and laser power.

\subsection{Particle removal mechanism}

Figure 4 presents the particle removal process. The process can be divided into three stages: (a) initial slight removal by shock waves, (b) continuous removal by bubble flow, and (c) rapid removal by combining bubbles. In the initial shock wave stage, when the laser is applied to the wafer surface, shock waves are initiated in the wafer, which remove some particles from the wafer surface. In the second stage, the bubble flow induced from the acoustic streaming removes more particles than those removed in the first stage. The bubbles and water rise up and jet onto the wafer surface, thus causing the particles to drop off the wafer surface. In the third stage, the bubbles cluster on the wafer surface and combine together. The bubbles' expelled effect from the wafer's center towards the outside across the surface area causes the particles to drop off the wafer surface rapidly.

\section{Experiments of laser cleaning}

\subsection{Laser stationary cleaning}

The single-point cleaning was performed as shown in Fig. 1. The laser was applied to the backside of the wafer. The laser power ranged from 20 to $60 \mathrm{~W}$ at a repetition rate of $20 \mathrm{kHz}$ and a pulse width of $150 \mathrm{~ns}$. The focal plane was placed $40 \mathrm{~mm}$ above the wafer surface. The images of the wafer after cleaning at a laser power of 25, 35, and $60 \mathrm{~W}$ are presented in Fig. 5. Cleaning experiments were performed on each wafer with a laser application time ranging from 1 to $7 \mathrm{~s}$. The white region depicts the alumina particles, and the circular gray dark region depicts the particle removal region.

The optical microscopy image of the cleaning area for a laser application time of $5 \mathrm{~s}$ and a laser power of $35 \mathrm{~W}$ is presented in Fig. 5. The particle removal area was circular in shape and was free of any alumina particles. The diame-
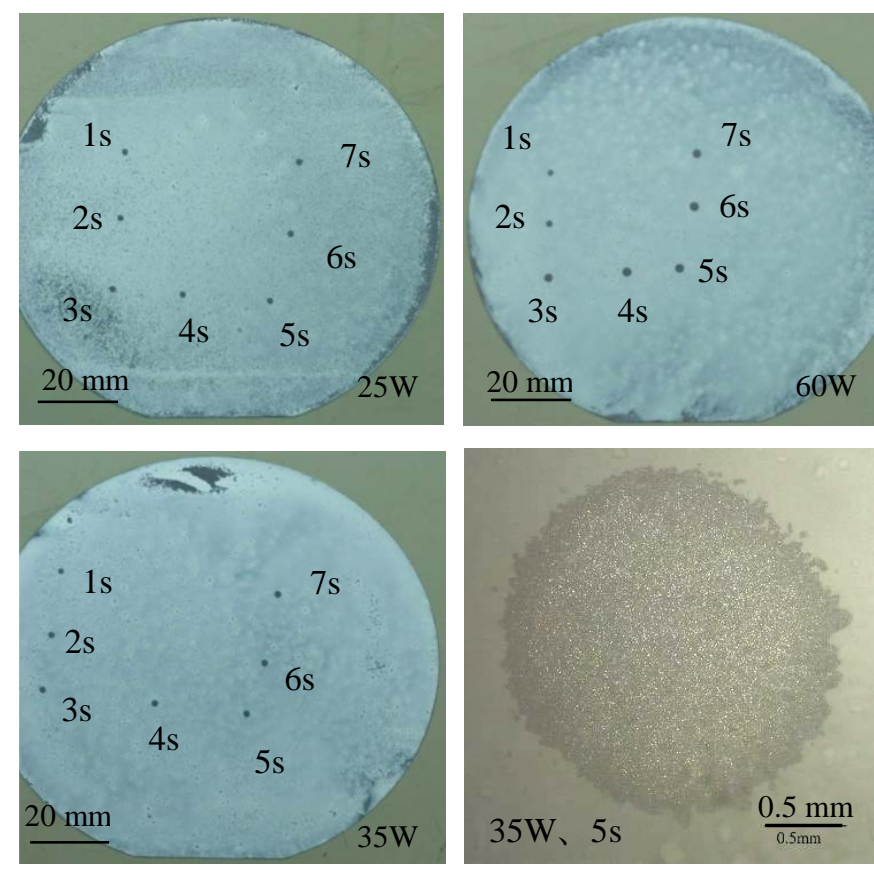

Fig. 5 Images of the particle removal areas by laser stationary cleaning.

ter of the particle removal region was approximately 1.83 $\mathrm{mm}$, and the area was $2.63 \mathrm{~mm}^{2}$.

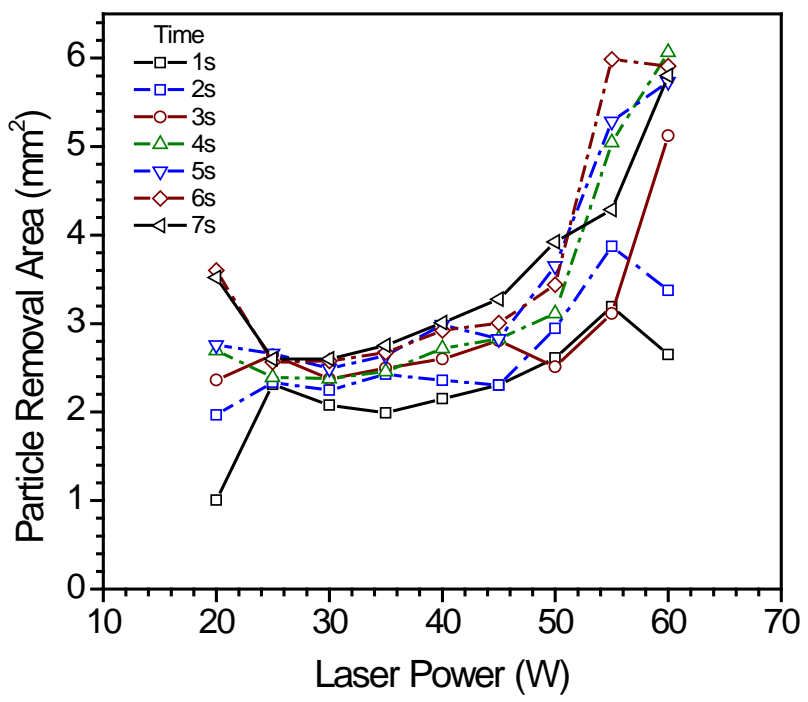

Fig. 6 Particle removal areas by laser stationary cleaning. 


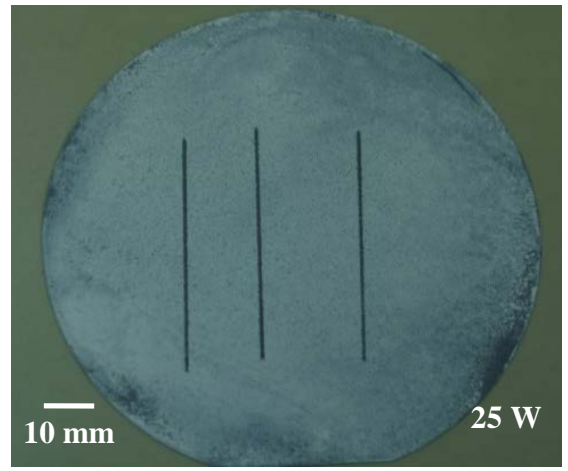

(a)

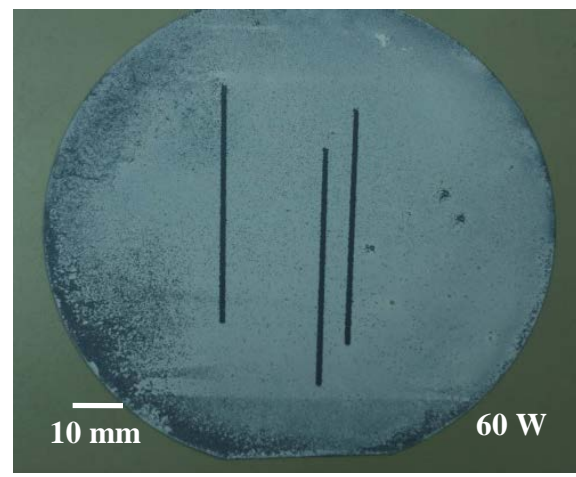

(b)

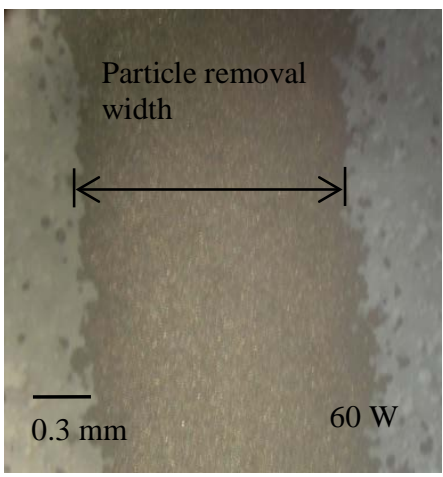

(c)

Fig. 7 Images of the particle removal areas by laser scan cleaning with scanning speed of $10 \mathrm{~mm} / \mathrm{s}$.

The relationship between laser power and the particle removal area at the seven cleaning time durations are presented in Fig. 6. In general, the higher the laser power is, the larger the particle removal area is. For a laser output of $20 \mathrm{~W}$ at a cleaning time of $1 \mathrm{~s}$, a particle area of $1 \mathrm{~mm}^{2}$ was removed. After a cleaning time of $3 \mathrm{~s}$, the particle removal area increased to $2.36 \mathrm{~mm}^{2}$. For a laser power of $60 \mathrm{~W}$, the removal area rapidly increased to $5.12 \mathrm{~mm}^{2}$ by $3 \mathrm{~s}$ of cleaning.

Because the laser energy can enhance the movement of the bubble, the particle removal increases with laser power. This phenomenon can be observed in the images captured during the bubble generation process. The particle removal occurred continuously during the laser application process, and increased with irradiation time.

The rolationships between the laser power and particle removal area as presented in Fig. 6 were obtained at a constant laser spot diameter of $1.69 \mathrm{~mm}$. The substrate subjected to the defocused laser energy was free from any melting effect. The experimental results demonstrated no obvious relationship between the laser power density and particle removal area at a constant laser power.

\subsection{Laser scan cleaning}

Laser scan cleaning was performed by applying the laser to the wafer moves in a straight line at a constant speed. The laser power used ranged from 30 to $60 \mathrm{~W}$, and the repetition rate was $20 \mathrm{kHz}$. The laser scanning speed ranged from 1 to $20 \mathrm{~mm} / \mathrm{s}$.

Laser cleaning at a laser power of 25 and $60 \mathrm{~W}$ and a scanning speed of $10 \mathrm{~mm} / \mathrm{s}$ are presented in Fig. 7(a) and 7(b), respectively. The optical microscopy image of the cleaning area at a laser power of $60 \mathrm{~W}$ is presented in Fig. 7(c). The surface was free from any alumina particles.

The particle removal width was measured to evaluate the cleaning performance. The relationship among the laser power, scanning speed, and particle removal width is presented in Fig. 8. For a scanning speed of $1 \mathrm{~mm} / \mathrm{s}$ and a laser power of $30 \mathrm{~W}$, the particle removal width was $1.50 \mathrm{~mm}$, which increased to $1.89 \mathrm{~mm}$ at a laser power of $60 \mathrm{~W}$. For a scanning speed of $20 \mathrm{~mm} / \mathrm{s}$, the particle removal widths were $0.76 \mathrm{~mm}$ and $1.20 \mathrm{~mm}$ for the laser powers of 30 and $60 \mathrm{~W}$, respectively.

Because the laser energy irradiated on the silicon surface for slow scanning is higher than that for fast scanning, the particle removal efficiency can be increased by decreasing the laser scanning speed. The laser scanning speed cor- relates with the particle removal performance. Thus, the slower the scanning speed is, the larger the particle removal width is.

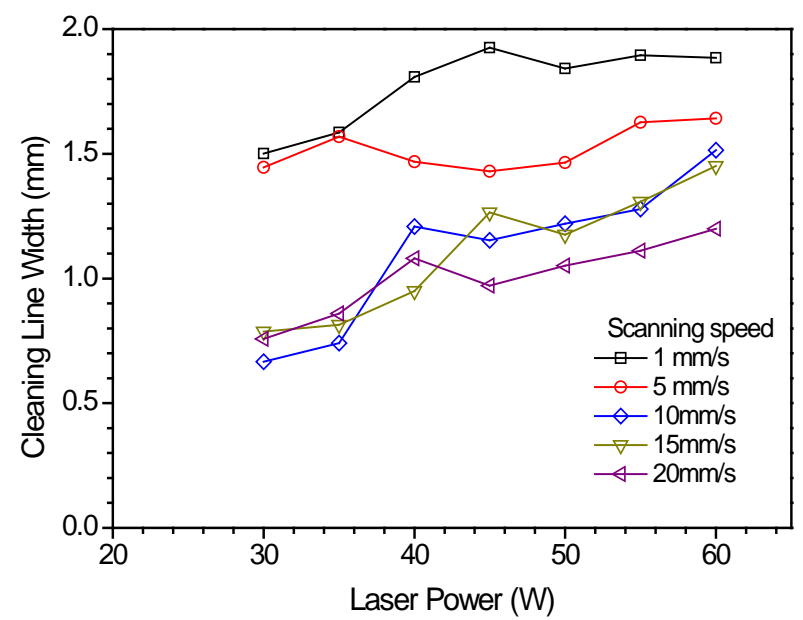

Fig. 8 Particle removal widths of laser scan cleaning.

\subsection{Temperature}

Water cooling can prevent the melting of the silicon wafer and alumina particles. The increase in temperature at the surface of the silicon wafer subjected to the Nd:YAG laser was measured using a thermocouple with an electronic data acquisition system. The temperature was recorded at $1 \mathrm{~s}$ intervals. The thermocouple accuracy was $\pm 0.5^{\circ} \mathrm{C}$. The temperatures at the laser application positions in the wet cleaning process are presented in Fig. 9. In this method, the silicon wafer was placed on the water surface, and the laser focal plane was located above the silicon wafer surface at a distance of $40 \mathrm{~mm}$. The initial temperature at the top surface of the wafer was $18.1^{\circ} \mathrm{C}$. When an $\mathrm{Nd}$ :YAG laser of $40 \mathrm{~W}$ power was applied on the wafer surface, the temperature at the top surface of the wafer increased to $47.3^{\circ} \mathrm{C}$ within $7 \mathrm{~s}$. Because the increase in temperature was small during the laser cleaning process, thermal effect may be ignored.

\section{Conclusions}

The laser cleaning technique that uses laser-induced acoustic streaming for silicon wafers was successfully demonstrated to remove submicron particles of size 0.5 $\mu \mathrm{m}$. The Nd:YAG laser applied from the back side of the wafer induced shock waves in the wafer. The shock waves 
were transmitted into the water, generating an acoustic streaming that removes the alumina particles from the wafer surface. The cleaning efficiency of this new method was excellent, and the laser power required was less than $50 \mathrm{~W}$. The temperature increase in the wafer caused by the laser heating was less than $30^{\circ} \mathrm{C}$. Therefore, the proposed technique can prevent thermal damage and has great potential to provide an improved solution for wafer cleaning.

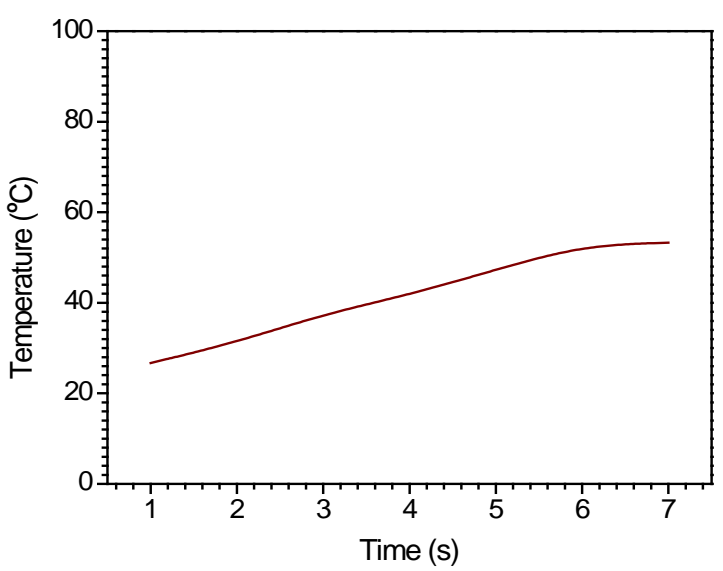

Fig. 9 Surface temperature of silicon wafers subjected to an Nd:YAG laser.

\section{Acknowledgments}

The authors gratefully acknowledge the financial support of this research by the National Science Council (Taiwan) under Grant NSC 100-2221-E-211-006 to Huafan University.

\section{References}

[1] W.L. Nyborg, J. Acoust. Soc. Am. 25, (1953) 68.

[2] S.J. Lighthill, J. Sound Vib. 61, (1978) 391.

[3] S.K. R.S. Sankaranarayanan, S. Cular, V.R. Bhethanabotla, B. Joseph, Phys. Rev. E 77, (2008) 066308.

[4] K. Bakhtari, R.O. Guldiken, P. Makaram, A.A. Busnaina, J.G. Park, J. Electrochem. Soc. 153 , (2006) G846.

[5] V. Dobler, R. Oltra, J.P. Boquillon, M. Mosbacher, J. Boneberg, P. Leiderer, Appl. Phys. A 69, (1999) 335.

[6] Y.F. Lu, Y.W. Zheng, W.D. Song, J. Appl. Phys. 87, (2000) 1534.

[7] J.M. Lee, K.G. Watkins, J. Appl. Phys. 89, (2001) 6496.

[8] K. Imen, S.J. Lee, S.D. Allen, Appl. Phys. Lett. 58, (1991) 203.

[9] A. Kruusing, Opt. Laser Eng. 41, (2004) 307.

[10] Y.F. Lu, Y. Zhang, Y.H. Wan, W.D. Song, Appl. Sur. Sci. 138, (1999) 140.

[11] D. Grojo, M. BoyoMo-Onana, A. Cros, Ph. Delaporte, Appl. Sur. Sci. 252, (2006) 4786.

[12] D. Grojo, A. Cros, Ph. Delaporte, M. Sentis, Appl. Sur. Sci. 253, (2007) 8309.

[13] V.B. Bregar, J. Mozina, Appl. Phys. A 77, (2003) 633.

[14] T. Kim, J.M. Lee, S.H. Cho, T.H. Kim, Opt. Lasers Eng. 43, (2005) 1010.
[15] J.K. Park, J.W. Yoon, K.H. Whang, Appl. Phys. A 108, (2012) 269.

[16] J.K. Park, J.W. Yoon, S.H. Cho, Appl. Sur. Sci. 258, (2012) 6379.

[17] M. Mosbacher, V. Dobler, J. Boneberg, P. Leiderer, Appl. Phys. A 70, (2000) 669.

[18] F. Lang, M. Mosbacherp, P. Leiderer, Appl. Phys. A 77, (2003) 117.

[19] M. Mosbacher, H.J. Munzer, J. Zimmermann, J. Solis, J. Boneberg, P. Leiderer, Appl. Phys. A 72, (2001) 41.

[20] P. Leiderer, M. Olapinski, M. Mosbacher, J. Boneberg, Proc. SPIE 6261, (2006) 62610F.

[21] T. Geldhauser, F. Ziese, F. Merkt, A. Erbe, J. Boneberg, P. Leiderer, Appl. Phys. A 89, (2007) 109.

[22] H.J. Yi, S.K. Chae, United States Patent, Patent No.7,556,712B (2009)

(Received: June 20, 2016, Accepted: October 11, 2016) 